\title{
A Perturbação do Espetro do Autismo na Primeira Infância: O Modelo do Centro de Estudos do Bebé e da Criança de Avaliação Diagnóstica e Intervenção Terapêutica
}

\author{
Autism Spectrum Disorder in Infancy and Early Childhood: \\ The Model of the Centro de Estudos do Bebé e da Criança \\ for Diagnosis and Therapeutic Intervention
}

\author{
Cristina MARTINS HALPERN $\otimes^{1}$, Pedro CALDEIRA DA SILVA ${ }^{2}$, Diana COSTA ${ }^{1}$, Maria João NASCIMENTO², \\ Joana MESQUITA REIS², Maria Teresa MARTINS², Berta PINTO FERREIRA², Isabel SANTOS ${ }^{1}$, Lília CARVALHO², \\ Madalena PAIVA GOMES ${ }^{2}$, Manuela MARTINS ${ }^{1}$, Maria João PIMENTEL ${ }^{1}$, Patrícia LOPES ${ }^{1}$, Paula SILVA ${ }^{1}$, \\ Rita RAPAZOTE ${ }^{2}$, Sílvia CATARINO², Susana AIRES PEREIRA ${ }^{1}$, Susana PEREIRA², Sílvia AFONSO' \\ Acta Med Port 2021 Oct;34(10):657-663 - https://doi.org/10.20344/amp.13397
}

\section{RESUMO}

Introdução: O Centro de Estudos do Bebé e da Criança do Hospital Dona Estefânia desenvolveu um modelo multidisciplinar de atuação na suspeita de perturbação do espetro do autismo na primeira infância, aplicando a recente norma da Direção Geral da Saúde. Pretende-se descrever a sua apresentação e casuística.

Material e Métodos: Estudo retrospetivo descritivo da série de crianças(< 3 anos), observadas por suspeita de perturbação do espetro do autismo, entre janeiro de 2018 e setembro de 2019, segundo este modelo e a classificação DC:0-5 $5^{\text {TM }}$.

Resultados: Foram observadas 178 crianças. A idade média da primeira consulta foi de 27 meses. Do total de crianças observadas, 116 concluíram as sessões diagnósticas (diagnóstico eixo I): perturbação do espetro do autismo/ perturbação do espetro do autismo atípica precoce $(36 \%)$, perturbação do desenvolvimento da linguagem (18\%), outros(19\%). Em $26 \%$ dos casos, o quadro foi atribuído a fatores classificados em outros eixos.

Discussão: O diagnóstico de perturbação do espetro do autismo foi colocado em $36 \%$, demonstrando o desafio diagnóstico das perturbações do neurodesenvolvimento na primeira infância. A casuística demonstra ainda que as características da relação com o cuidador (eixo II), a presença de condições físicas (eixo III), fatores de stress psicossociais (eixo IV) e a trajetória de desenvolvimento (eixo V) têm um impacto clínico significativo. É desejável a antecipação da idade de sinalização pelo impacto no prognóstico.

Conclusão: Este modelo é pioneiro em Portugal ao propor uma atuação conjunta de duas especialidades na primeira infância: pedopsiquiatria e neuropediatria/pediatria desenvolvimento. Este modelo de atuação melhora a acuidade diagnóstica e permite a intervenção terapêutica precoce.

Palavras-chave: Criança; Lactente; Perturbação do Espetro do Autismo/diagnóstico; Perturbação do Espetro do Autismo/tratamento; Portugal

\section{ABSTRACT}

Introduction: The Centro de Estudos do Bebé e da Criança in Hospital Dona Estefânia has organized a multidisciplinary model for children under three with suspected autism spectrum disorder, thus implementing the recent guidelines established by the Directorate General for Health. The aim of this study is to describe this model and case series.

Material and Methods: A retrospective descriptive study of observed children with suspected ASD. They were observed according to the model of the Centro de Estudos do Bebé e da Criança and DC:0-5 ${ }^{\mathrm{TM}}$ classification, between January 2018 and September 2019.

Results: The study included 178 children. The average age at the initial assessment was 27 months. From the total sample, 116 children concluded the diagnostic sessions (axis I): Autism Spectrum Disorder/Early Atypical (36\%), Developmental Language Disorder (18\%), Other (19\%). Factors of axes II, III, IV and V of DC:0-5 $5^{\mathrm{TM}}$ were determinant for clinical diagnosis in $26 \%$.

Discussion: Of 116 children, 36\% were diagnosed with Autism Spectrum Disorder. This highlights the diagnostic challenge posed by neurodevelopmental disorders in early infancy. The sample shows that the characteristics of the relationship with the caregiver (axis II), presence of physical conditions (axis III), psycho-social stressors (axis IV) and developmental trajectory (axis V) have a significant clinical impact. In the future, the initial assessment should take place well before the age of 27 months because of the impact on prognosis. Conclusion: This model is a pioneering approach in Portugal. It promotes a common approach of Child and Adolescent Psychiatry and Neuropediatrics/Developmental Pediatrics in early infancy. Moreover, it increases the diagnostic acuity of Autism Spectrum Disorders and early therapeutic intervention.

Keywords: Autism Spectrum Disorder/diagnosis; Autism Spectrum Disorder/therapy; Child; Infant; Portugal

\section{INTRODUÇÃO}

A perturbação do espetro do autismo (PEA) constitui, na verdade, um grupo heterogéneo de perturbações do neurodesenvolvimento que se caracterizam globalmente por um comprometimento da interação social e da comunicação (verbal e não verbal) e pela existência de padrões de comportamentos repetitivos e/ou um leque restrito de

1. Centro de Estudos do Bebé e da Criança. Unidade de Desenvolvimento. Hospital Dona Estefânia. Centro Hospitalar Universitário de Lisboa Central. Lisboa. Portugal.

2. Centro de Estudos do Bebé e da Criança. Unidade da Primeira Infância da Especialidade de Psiquiatria da Infância e da Adolescência. Hospital Dona Estefânia. Centro

Hospitalar Universitário de Lisboa Central. Lisboa. Portugal.

$\triangle$ Autor correspondente: Cristina Martins Halpern. cristinamhalpern@gmail.com

Recebido: 02 de janeiro de 2020 - Aceite: 19 de outubro de 2020 - First published: 27 de janeiro de 2021 - Online issue published: 01 de outubro de 2021 Copyright $\odot$ Ordem dos Médicos 2021 
interesses. De início precoce na infância, a PEA pode condicionar limitações importantes no funcionamento emocional e social da criança, com dificuldades que podem persistir ao longo do tempo. ${ }^{1-3}$

A intervenção terapêutica precoce tem fortes implicações na evolução e no prognóstico da PEA. ${ }^{4-10}$ Sabemos hoje que a adoção de uma intervenção terapêutica de base relacional, que tenha em consideração os avanços das neurociências e o reconhecimento da importância das interações entre os genes e o ambiente para a organização neuronal, possibilita evoluções muito positivas, demonstrando que nem sempre o curso desta perturbação é crónico e de mau prognóstico. .,6-14 $^{-1}$

Em crianças muito pequenas, o diagnóstico de PEA é um desafio clínico difícil, não só pelos aspetos relacionados com o desenvolvimento, mas também pela instabilidade dos sintomas nesta idade. .,4,9 $^{3}$ compreensão e a sistematização das especificidades da PEA e de todo o grupo de doenças do neurodesenvolvimento e da saúde mental na primeira infância requer uma classificação que proponha uma abordagem multiaxial destas perturbações, e que permita, de facto, estabelecer o diagnóstico e discutir os diagnósticos diferenciais. . $^{3,4,15,16}$

Este é o motivo principal para a adoção da classificação diagnóstica das perturbações da saúde mental e do desenvolvimento da infância (0-5) DC:0-5 Diagnostic Classification of Mental Health and Developmental Disorders of Infancy and Early Childhood"(tradução portuguesa) e não de outras classificações, como a DSM 5 Diagnostic and Statistical Manual of Mental Disorders e a ICD-10 International Statistical Classification of Diseases and Related Health Problems, que são usualmente utilizadas para outras faixas etárias e insuficientes na primeira infância. ${ }^{17-19}$

Quanto ao diagnóstico de PEA, a classificação DC:0$-5^{\mathrm{TM}}$ inclui a perturbação do espetro do autismo tal como a DSM 5 e a ICD-10, mas contempla também uma nova entidade, a perturbação do espetro do autismo atípica precoce (PEAAP), que permite identificar sinais precoces de PEA em crianças com idades compreendidas entre os nove e os 36 meses de idade. ${ }^{3,17}$

Para o diagnóstico de PEAAP, a criança deverá apresentar pelo menos duas dificuldades ao nível da comunicação/socialização e pelo menos um critério de comportamento restrito repetitivo com algum nível de comprometimento funcional. Assim, comparativamente com o proposto para o diagnóstico de PEA, o diagnóstico de PEAAP requer a presença de um menor número de critérios clínicos numa idade mais precoce, o que permite a identificação de dificuldades que podem ser alvo de intervenção desde muito cedo na vida. ${ }^{17}$

Além dos critérios de diagnóstico das perturbações clínicas descritos no eixo I da DC:0-5 ${ }^{\mathrm{TM}}$, a abordagem multiaxial desta classificação considera também: no eixo II, as questões referentes ao contexto relacional; no eixo III, as condições e considerações da saúde física; no eixo IV, os fatores de stress psicossociais e; no eixo $V$, as competências e etapas do desenvolvimento. Em cada um dos eixos, para além de identificar as perturbações e as vulnerabilidades, é também possível encontrar as forças que podem potenciar a intervenção. Assim, a caracterização de cada eixo tem um impacto clínico importante e deve ser especificamente considerada no diagnóstico, no tratamento e na compreensão da trajetória de evolução das crianças.

Ao contrário da maioria das doenças para as quais existe um seguimento e tratamento específico já bem estabelecido, apenas recentemente estão a surgir normas de orientação clínica para a PEA que considerem as diferentes faixas etárias, ${ }^{20-25}$ como por exemplo a norma de orientação clínica (NOC) da Direção Geral de Saúde (DGS) denominada "Abordagem diagnóstica e Intervenção na Perturbação do Espetro do Autismo em Idade Pediátrica e no Adulto", publicada em abril de 2019. ${ }^{26}$

Esta norma propõe a uniformização da abordagem da PEA a nível nacional, clarificando os aspetos relativos à identificação precoce, avaliação diagnóstica e intervenção terapêutica. Especificando um pouco quanto à identificação precoce e avaliação diagnóstica, a norma prevê que seja feita a referenciação para uma consulta de especialidade hospitalar em crianças com suspeita de PEA, onde a decisão diagnóstica, a intervenção terapêutica, e o seguimento, devem envolver uma equipa multidisciplinar. Esta referenciação de crianças dos 0 - 3 anos obriga a que a primeira consulta seja realizada num prazo máximo de 30 dias. ${ }^{26}$

A equipa deve ter formação e experiência na PEA e incluir um pediatra com experiência na área do neurodesenvolvimento e/ou neuropediatra e um psiquiatra da infância e adolescência. Considera ainda necessário o envolvimento de psicólogo, enfermeiro, assistente social, terapeuta da fala e terapeuta ocupacional ou técnico superior de educação especial e reabilitação. Deverá ser efetuada uma abordagem individualizada de cada criança, que deve assentar na terapêutica não farmacológica de base relacional. ${ }^{26}$

Para dar resposta a estas diversas dimensões clínicas, cumprir a NOC da DGS e os prazos estabelecidos, o Centro de Estudos do Bebé e da Criança (CEBC) do Hospital Dona Estefânia - Centro Hospitalar Universitário de Lisboa Central (CHLC) organizou um modelo multidisciplinar de avaliação diagnóstica e intervenção para as crianças dos 0 aos 3 anos com suspeita de perturbação do espetro do autismo.

Inaugurado em 2017, o CEBC congrega a Unidade da Primeira Infância da Especialidade de Psiquiatria da Infância e da Adolescência e a Unidade de Desenvolvimento do Hospital Dona Estefânia. Destina-se sobretudo ao atendimento diferenciado de crianças até aos cinco anos, observando de forma prioritária crianças até aos três anos de idade. Nas crianças até aos três anos de idade em que sejam identificadas preocupações quanto ao desenvolvimento e saúde mental, o contacto com o CEBC pode ser direto por parte dos pais ou por referenciação médica.

\section{MATERIAL E MÉTODOS}

Estudo retrospetivo descritivo da série de casos de crianças com suspeita de PEA na primeira infância (0 - 3 
anos) observadas segundo o modelo de atuação do CEBC de avaliação diagnóstica e intervenção terapêutica.

Tratando-se da apresentação e descrição de um modelo de diagnóstico, não foram solicitados consentimentos informados específicos para este estudo. No entanto considera-se que pode ser enquadrado na análise de dados de saúde para efeitos de diagnóstico e melhoria de cuidados. Os dados foram recolhidos por consulta do processo clínico. Foram incluídas todas as crianças até aos três anos de idade sinalizadas por suspeição de PEA entre janeiro de 2018 e setembro de 2019.

Os dados pessoais foram codificados de forma a garantir a confidencialidade e a privacidade do participante. O estudo foi aprovado pela Comissão de Ética do Centro Hospitalar Universitário Lisboa Central.
A equipa técnica do CEBC é constituída por três pedopsiquiatras, uma neuropediatra, três pediatras, duas psicólogas clínicas, duas enfermeiras especialistas em saúde mental e psiquiatria, duas enfermeiras especialistas em saúde infantil e pediatria, uma terapeuta ocupacional, uma terapeuta da fala, uma assistente social e uma professora de educação especial.

O modelo CEBC de avaliação diagnóstica e intervenção para as crianças dos 0 aos 3 anos com suspeita de perturbação do espetro do autismo prevê (Fig. 1):

1. avaliação inicial em consulta de desenvolvimento e pedopsiquiatria por médicos especialistas e enfermeiras especialistas em saúde mental e psiquiatria e em saúde infantil e pediatria;

\section{Suspeita de perturbação do espetro do autismo}

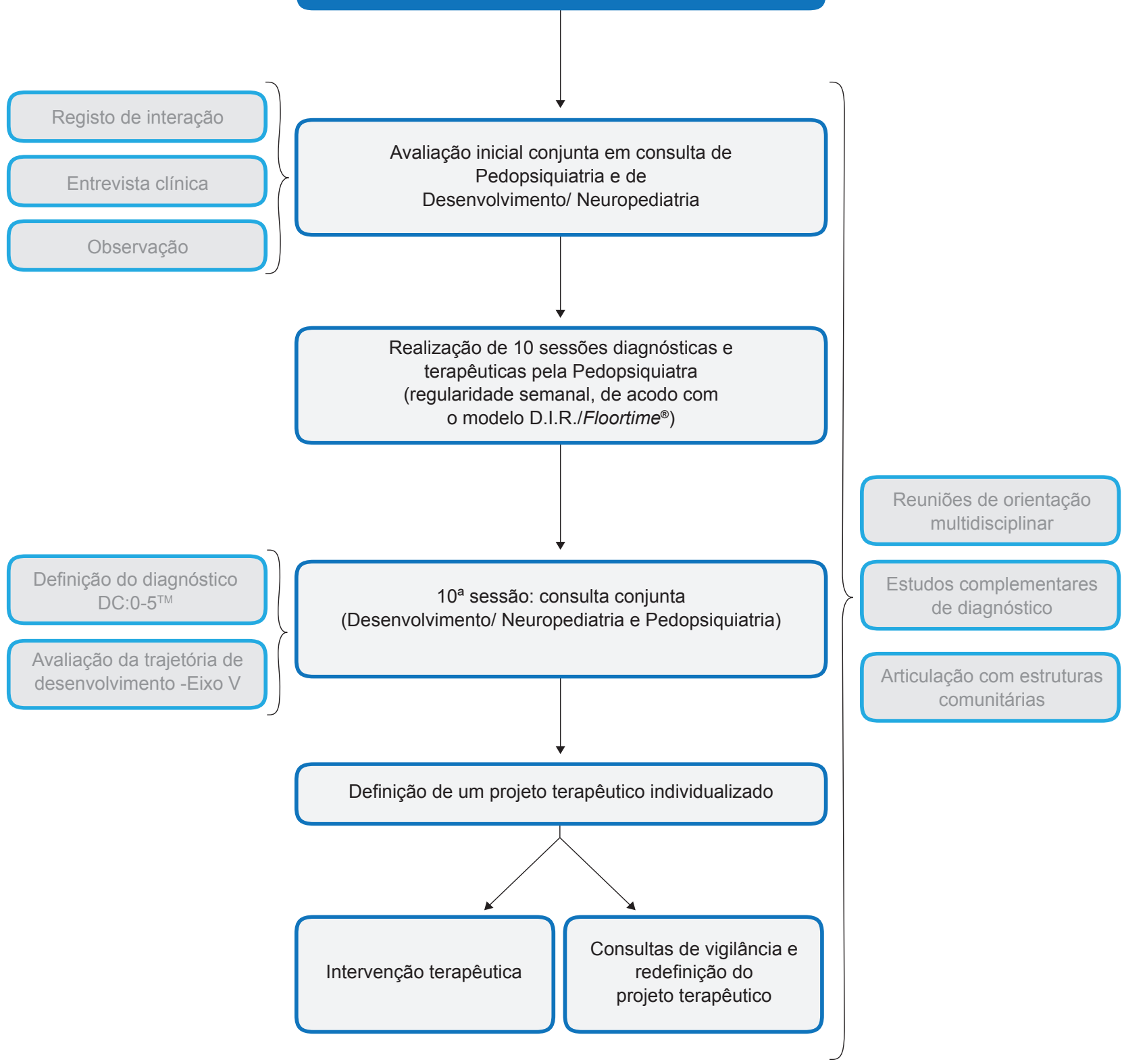

Figura 1 - O modelo do CEBC de avaliação diagnóstica e intervenção terapêutica nas perturbações do espetro do autismo na primeira infância 
2. seguimento em 10 sessões diagnósticas de pedopsiquiatria com regularidade semanal. Estas sessões visam esclarecer o diagnóstico diferencial e iniciar uma intervenção terapêutica de base relacional, baseada no modelo D.I.R./Floortime ${ }^{\circledR}$ (modelo baseado no desenvolvimento, nas diferenças individuais e na relação), adotado como modelo de eleição para o tratamento da PEA ${ }^{6,9,14,27}$; desta forma abordam-se os eixos I, II, IV;

3. a décima sessão diagnóstica é uma consulta conjunta envolvendo pedopsiquiatras e neuropediatra/ pediatras do desenvolvimento; é nesta etapa que se estabelece o quadro final, considerando os aspetos referentes à evolução clínica, diagnóstico diferencial, condições e considerações da saúde física (eixo III da classificação DC:0-5 ${ }^{\mathrm{TM}}$ ), necessidade de esclarecimento e investigação complementar e as competências do desenvolvimento (eixo $\mathrm{V}$ da classificação DC:0-5 ${ }^{\mathrm{TM}}$ );

4. intervenção da Terapia Ocupacional, Psicologia ou Terapia da Fala e articulação com as estruturas comunitárias, como as creches ou jardins infantis e as equipas locais de intervenção precoce (Sistema Nacional de Intervenção Precoce na Infância);

5. discussão diagnóstica e orientação terapêutica alargada a toda a equipa presente em reuniões clínicas semanais (reuniões de observação da interação, com suporte de registos vídeo da primeira consulta e reuniões de orientação clínica);

6. identificação de um profissional de referência (médico, enfermeira e/ou psicóloga, terapeuta ocupacional ou terapeuta da fala), que, após as sessões diagnósticas, passa a ser o elemento de ligação que acompanhará cada caso.

\section{RESULTADOS}

Neste período de tempo foram observadas 178 crianças com idade inferior a três anos e com suspeita de PEA, dos quais 135 (76\%) eram do sexo masculino.

Os pedidos de consulta tiveram origem maioritariamente no médico de família ( $n=69 ; 39 \%$ ) e no pedido direto das famílias $(n=48 ; 27 \%)$. A referenciação a partir de outras unidades do Hospital Dona Estefânia representou 34\% dos casos $(n=61)$, distribuindo-se pelas especialidades de Pediatria Médica (13\%), Neuropediatria (7\%), consulta de risco de Neurodesenvolvimento (4\%), Serviço de Urgência (3\%) e os restantes $7 \%$ distribuíram-se por outras especialidades.

As preocupações mais frequentemente referidas pelos médicos assistentes e pelas famílias foi o atraso na aquisição da linguagem e/ou preocupações quanto a competências de comunicação social /interação e/ou a indicação expressa de suspeita de perturbação do espetro do autismo $(\mathrm{n}$ total $=160)$. As restantes preocupações prendiam-se com perturbações do comportamento, como birras ou hipercinésia, perturbações do sono e da alimentação, que no primeiro contacto com o CEBC foram valorizadas num contexto mais amplo de suspeita de PEA.

A primeira consulta ocorreu entre os nove e os 36 meses, com uma média de 27 meses, observando-se um aumento gradual dos casos referenciados a partir dos nove meses, atingindo um primeiro pico aos 23 meses $(n=12)$ e um segundo pico aos 35/36 meses $(n=26)$.

Em 25\% dos casos estudados ( $n=44)$, observou-se que ambos os pais são imigrantes provenientes do Nepal $(n=13)$, Brasil $(n=13)$, Angola $(n=7)$, Bangladesh $(n=4)$, Ucrânia $(n=3)$, São Tomé e Príncipe $(n=2)$, Índia $(n=1)$ e Roménia $(n=1)$.

Dos 178 casos observados, procedeu-se à discussão diagnóstica de 116; as restantes crianças mantêm-se ainda em sessões diagnósticas.

Foram propostos os seguintes diagnósticos $(n=116)$ : PEA $n=30$ e PEAAP $n=12$ (total $36 \%$ ), perturbação do desenvolvimento da linguagem $n=21(18 \%)$, atraso global do desenvolvimento psicomotor $n=12(10 \%)$, outros $n=11$ (9\%) (exemplo: perturbação pós-stress traumático, perturbação do humor, perturbação da integração sensorial).

Há a referir que, dos 116 casos discutidos, 30 crianças (26\%) não cumpriram critérios de diagnóstico para perturbações do neurodesenvolvimento (referentes ao eixo I da classificação DC:0-5 ${ }^{\mathrm{TM}}$ ), considerando-se assim como principais determinantes para o quadro os fatores classificados, respetivamente, nos eixos II, IV e V da DC:0-5 $5^{\mathrm{TM}}$ (referentes ao contexto relacional; ao stress psicossocial; e a trajetórias individuais de desenvolvimento).

Após a discussão diagnóstica multidisciplinar $(n=116)$, foi realizado um plano terapêutico que incluiu consultas de vigilância e terapêuticas na Unidade da Primeira Infância (40\%) e na Unidade de Desenvolvimento (42\%; destes últimos, $8 \%$ em sessões individuais de promoção do desenvolvimento). No decorrer do seguimento, $13 \%(n=15)$ dos casos foram transferidos para unidades da área de residência e a totalidade foi referenciada às equipas locais de intervenção precoce.

Uma vez terminadas as sessões diagnósticas, foi obtido apoio terapêutico regular de Terapia da Fala para 40 crianças (34\%), Terapia Ocupacional para 17 crianças (15\%), e Psicologia para três crianças, tendo-se mantido o acompanhamento regular pelo CEBC. Cinco crianças iniciaram planos terapêuticos intensivos como por exemplo o da Associação de Apoio à Unidade da Primeira Infância (AAUPI). (Tabela 1)

O estudo complementar para investigação etiológica e/ ou aconselhamento genético ao casal foi realizado em $25 \%$ dos casos $(n=45)$, incluindo referenciação a consulta de genética, estudo metabólico, estudo por neuroimagem e eletroencefalograma quando considerado adequado.

\section{DISCUSSÃO}

O diagnóstico diferencial da PEA nesta idade precoce implica o afastamento de outras perturbações de saúde mental e neurodesenvolvimento, para os quais é necessária a experiência técnica, a adoção de critérios e a consideração de eixos que não estão previstos na classificação 
Tabela 1 - Caracterização da população quanto ao diagnóstico e plano terapêutico

\begin{tabular}{|c|c|}
\hline Sexo & $n=178$ \\
\hline Feminino & $43(24 \%)$ \\
\hline Masculino & $135(76 \%)$ \\
\hline Origem do pedido de consulta & $n=178$ \\
\hline Médico de família & $69(39 \%)$ \\
\hline Pedido direto das famílias & $48(27 \%)$ \\
\hline Outros serviços do hospital & $61(34 \%)$ \\
\hline Diagnósticos atribuídos (nas crianças que concluíram as 10 sessões) & $n=116$ \\
\hline Perturbação do espetro do autismo & $30(26 \%)$ \\
\hline Perturbação do espetro do autismo atípica precoce & $12(10 \%)$ \\
\hline Perturbação do desenvolvimento da linguagem & $21(18 \%)$ \\
\hline Atraso global do desenvolvimento psicomotor & $12(10 \%)$ \\
\hline Outros diagnósticos & $11(9 \%)$ \\
\hline Sem diagnóstico de Eixo I & $30(26 \%)$ \\
\hline Plano terapêutico (nas crianças que concluíram as 10 sessões) & $n=116$ \\
\hline Consultas na Unidade da Primeira Infância & $46(40 \%)$ \\
\hline Consultas na Unidade de Desenvolvimento & $48(42 \%)$ \\
\hline Transferências para as Unidades da área de residência & $15(13 \%)$ \\
\hline Intervenção pelas equipas locais de intervenção precoce & $116(100 \%)$ \\
\hline Terapia da Fala & $40(34 \%)$ \\
\hline Terapia Ocupacional & $17(15 \%)$ \\
\hline Psicologia & $3(3 \%)$ \\
\hline Intervenção multidisciplinar na Associação de Apoio à Unidade da Primeira Infância & $5(4 \%)$ \\
\hline
\end{tabular}

DSM 5 e que constituem o cerne da abordagem do CEBC.

A nossa casuística documenta que das 116 crianças que completaram a avaliação diagnóstica por suspeita de PEA, apenas $36 \%$ cumprem critérios diagnósticos de PEA/ PEAAP (eixo I DC:0-5 ${ }^{\mathrm{TM}}$ ), o que nos alerta para a dificuldade diagnóstica desta perturbação.

Por outro lado, em cerca de $26 \%$ dos casos de crianças sinalizadas por suspeita de PEA não atribuímos nenhum diagnóstico quanto a perturbações do neurodesenvolvimento (eixo I DC:0-5 $5^{\mathrm{TM}}$ ). Este dado é relevante, pois alerta para o impacto dos elementos considerados nos outros eixos da classificação, nomeadamente as características da relação com o cuidador (eixo II), a presença de condições físicas (eixo III), os fatores de stress psicossociais (eixo IV) e a própria trajetória de desenvolvimento da criança, que pode apresentar desvios transitórios de rotas.

No decorrer das sessões diagnósticas iniciais e, mais tarde, no seguimento pelo CEBC, a análise da evolução de cada criança, apoiada pela avaliação da trajetória de desenvolvimento (eixo $\mathrm{V} D \mathrm{DC}: 0-5^{\mathrm{TM}}$ ), é importante na ponderação e racionalização dos exames complementares de diagnóstico pedidos. A investigação complementar foi realizada em cerca de $25 \%$ dos casos, considerando a presença de regressão do desenvolvimento, alterações ao exame somático ou sinais neurológicos focais, irritabilidade ou apatia extrema, ausência de evolução clínica favorável ou estagnação durante as sessões, condições co-mórbidas como epilepsia, o envolvimento global das várias competências de desenvolvimento ou o desejo do casal de aconselhamento genético. $26,28,29$

De referir que o plano de seguimento no CEBC passa por uma orientação pelo médico e técnico de referência e, consoante a evolução clínica, este seguimento pode ser reformulado para que inclua diferentes valências do CEBC. $A$ intervenção terapêutica nos casos de PEA passou pela manutenção de sessões baseadas no modelo D.I.R/Floortime ${ }^{\circledR}$ e na progressiva articulação com a comunidade. Nos casos restantes, as intervenções foram discutidas caso a caso, dependendo das especificidades, das necessidades da família e dos recursos disponíveis na comunidade. Nalguns casos envolveram o apoio por enfermeiro especialista, psicólogo, terapeuta ocupacional e/ou terapeuta da fala. Globalmente, a distribuição do seguimento entre as unidades do CEBC (Unidade da Primeira Infância e a Unidade de Desenvolvimento) foi idêntica. Esta dinâmica flexível evita a sobreposição de atuações e de consultas e favorece a adesão das famílias, ao diminuir a sobrecarga familiar e o absentismo dos pais ao trabalho.

Consideramos, assim, que este modelo oferece uma resposta flexível e que se tem mostrado eficaz e eficiente. Nesta amostra de conveniência respondemos a um número muito significativo de pedidos $(n=178)$, recebidos num curto espaço de tempo (21 meses); a pedido das famílias, permitimos o acesso direto a cuidados muito especializados; 
e foram globalmente respeitados os prazos previstos na NOC da DGS para uma primeira consulta (menos de 30 dias).

Este modelo visa também privilegiar uma intervenção terapêutica precoce nas crianças com suspeita de PEA e o esperado impacto na melhoria do seu prognóstico. A precocidade da intervenção depende da idade média de observação na primeira consulta, que nesta amostra é de 27 meses. Pensamos que esta idade pode ainda ser antecipada, pois neste estudo foi identificado um pico de referenciação aos 23 meses, após um crescimento gradual desde os nove meses.

Esperamos que se acentue a tendência para uma referenciação ainda mais precoce que os 23 meses, uma vez que, por um lado, é possível reconhecer importantes sinais clínicos de suspeição de PEA no primeiro ano de vida e, por outro lado, o rastreio universal desta condição está previsto no Programa Nacional de Saúde Infantil e Juvenil da DGS para os 18 meses (através da aplicação do questionário M-CHAT / M-CHAT R/T). . ${ }^{30-32}$ Neste sentido, justifica-se o reforço de sensibilização dos pediatras, médicos de medicina geral e familiar, enfermeiros das equipas de cuidados de saúde primários e enfermeiros especialistas em pediatria e saúde infantil e saúde mental e pedopsiquiatria para uma referenciação mais atempada.

Sinalizamos também nesta casuística a presença de crianças cujos pais têm nacionalidade estrangeira, de origens muito diversas, multiculturais, representando um quarto dos casos observados. Este número espelha o facto do Hospital Dona Estefânia servir as freguesias do país com maior presença de multiculturalidade. ${ }^{33}$ Destas crianças, nove foram diagnosticadas com PEA/PEA atípica precoce. Não temos elementos no estudo que nos permitam, neste curto espaço de tempo, discutir a influência da imigração/multiculturalidade no surgimento de sinais de suspeita de PEA, nem a sua influência na evolução clínica das crianças. Contudo, a nossa reflexão é que a imigração e a multiculturalidade podem acentuar uma vulnerabilidade social, familiar e económica com reflexo nas características das relações estabelecidas e na presença de fatores de stress adicionais (eixos II e IV DC:0-5 $5^{\mathrm{TM}}$ ).

A intervenção terapêutica na PEA passa pelo acesso a intervenção direta e frequente em sessões de Terapia Ocupacional, Terapia da Fala e Psicologia, muitas vezes necessitando de duas ou três destas valências em simultâneo. ${ }^{6,14,27}$ Neste aspeto, pela escassez de recursos humanos conforme referido nos nossos resultados, há uma limitação nos cuidados públicos de Terapia da Fala, Ocupacional ou Psicologia, documentada no número de crianças na casuística que obtiveram estas intervenções de forma direta e regular, que consideramos baixo.

\section{CONCLUSÃO}

O modelo de atuação do CEBC de avaliação diagnóstica e intervenção terapêutica em crianças com suspeita de PEA na primeira infância (0 - 3 anos) é pioneiro e, tanto quanto sabemos, único em Portugal, ao promover a atuação conjunta, e muitas vezes simultânea, da equipa da Unidade da Primeira Infância da Especialidade de Psiquiatria da Infância e da Adolescência e da Unidade de Desenvolvimento. Pensamos que a atuação conjunta entre as especialidades e as competências previstas no modelo CEBC, o seguimento e a intervenção em sessões diagnósticas semanais e a discussão regular em equipa (incluindo a observação em registos gravados) contribuem para o rigor diagnóstico da PEA na primeira infância.

Na discussão diagnóstica, a adoção da DC:0-5 $5^{\mathrm{TM}}$, uma classificação diagnóstica multiaxial das perturbações de saúde mental e do desenvolvimento da primeira infância, permite considerar as especificidades deste grupo etário, discutir o diagnóstico diferencial e identificar áreas de intervenção nas forças e nas vulnerabilidades do funcionamento de cada criança e das relações estabelecidas. Além dos aspetos relacionados com a acuidade diagnóstica, este modelo de atuação do CEBC propõe ainda o acesso direto aos cuidados de saúde muito especializados, a pedido da família, o que: aumenta a acessibilidade e a equidade; permite a racionalização da investigação complementar; e promove desde logo a intervenção terapêutica precoce, já que cada uma das 10 sessões de pedopsiquiatria tem também um intuito terapêutico, ao ser pensada segundo o modelo D.I.R.

Este modelo favorece a organização dos recursos humanos da equipa: não duplica consultas, possibilita a complementaridade entre técnicos, e promove a articulação de cuidados com as estruturas comunitárias, aspeto este que se mostra muito relevante para o seguimento destas crianças a médio e longo prazo.

A inovação trazida pela criação recente desta estrutura única do Serviço Nacional de Saúde, o Centro de Estudos do Bebé e da Criança, e deste modelo de seguimento de crianças com idades entre os 0 e os 3 anos com suspeita de PEA, é o principal motivo desta reflexão. Este modelo, que integra a experiência de unidades com história e mérito reconhecidos, pode trazer importantes ganhos a curto e a médio prazo, ajudando também a repensar as estruturas locais necessárias para acompanhar estas crianças. ${ }^{34}$ Acreditamos que se tornará um modelo comprovadamente válido e possível de ser adotado por várias outras unidades com supervisão técnica inicial.

\section{CONTRIBUTO DOS AUTORES}

CMH, PCS, DC, MJN: Contribuição intelectual substancial, directa, no desenho e elaboração do artigo, análise e interpretação dos dados, redação do manuscrito, revisão de rascunhos, aprovação da versão final.

JMR, MTM: Contribuição intelectual substancial, directa, no desenho e elaboração do artigo, análise e interpretação dos dados, redação do manuscrito, aprovação da versão final.

BPF, IS, LC, MPG, MM, MJP, PL, PS, RR, SC, SAP, SP, SA: Contribuição intelectual substancial na revisão dos rascunhos, revisão crítica do conteúdo, aprovação da versão final. 


\section{PROTEÇÃO DE PESSOAS E ANIMAIS}

Os autores declaram que os procedimentos seguidos estavam de acordo com os regulamentos estabelecidos pelos responsáveis da Comissão de Investigação Clínica e Ética e de acordo com a Declaração de Helsínquia da Associação Médica Mundial actualizada em 2013.

\section{CONFIDENCIALIDADE DOS DADOS}

Os autores declaram ter seguido os protocolos do seu centro de trabalho acerca da publicação de dados.

\section{CONFLITOS DE INTERESSE}

Os autores declaram que não há conflito de interesses na publicação deste artigo.

\section{FONTES DE FINANCIAMENTO}

Os autores declaram não ter recebido subsídios ou bolsas para a elaboração do artigo.

\section{REFERÊNCIAS}

1. Couteur A, Szatmari P. Autism spectrum disorders. In: Thapar A, Pine $D$, Leckman J, Scott S, Snowling M, Taylor E, editors. Rutter's child and adolescent psychiatry. $6^{\text {th }}$ ed. Oxford: Wiley-Blackwell; 2018. p. 665-82

2. Ozonoff S, Rogers SJ, Hendren RL. Autism spectrum disorders: a research review for practitioners. Washington: American Psychiatric Publishing; 2003.

3. Barton M, Chen J. Autism spectrum disorder. In: Zeanah C, editor. Handbook of mental health. $4^{\text {th }}$ ed. New York: The Guildford Press; 2019. p. 308-23.

4. Caldeira da Silva P. Primeira infância. In: Monteiro P, editor. Psicologia e Psiquiatria da Infância e da Adolescência. Lisboa: Lidel; 2014.

5. MacDonald R, Parry-Cruwys D, Peterson P. Behavioral treatments. In: Hollander E, Hagerman R, Fein D, editors. Autism spectrum disorders. Washington: American Psychiatric Association; 2018. p.195-230.

6. Weider S, Foley G. The DIR model. In: Hollander E, Hagerman R, Fein D, editors. Autism spectrum disorders. Washington: American Psychiatric Association; 2018. p. 231-52.

7. Fein D, Barton M, Eigsti IM, Kelley E, Naigles L, Schultz RT, et al. Optimal outcome in individuals with a history of autism. J Child Psichol Psychiatry. 2013;54:195-205.

8. Gibb R, Kolb B, editors. The neurobiology of brain and behavioral development. London: Academic Press Editors; 2018.

9. Greenspan S, Wieder S. The child with special needs: encouraging intellectual and emotional growth. Reading: Perseus Books; 1998.

10. Gonzalez NM, Alpert M, Shay J, Campbell M, Small AM. Autistic children on follow-up: change of diagnosis. Psychopharmacol Bull. 1993;29:3538.

11. Dawson G, Rogers S, Munson J, Smith M, Greenson J, Donaldson A, et al. Randomized, controlled trial of an intervention for toddlers with autism: the early start Denver model. Pediatrics. 2010;125:e17-e23.

12 Pajareya K, Nopmaneejumruslers K. A pilot randomized controlled trial of DIR/FloortimeTM parent training intervention for pre-school children with autistic spectrum disorders. Autism. 2011;15:563-77.

13. Nelson B, Parker S, Siegel. Interpersonal neurobiology, mindsight and integration. In: Brandt K, Perry B, Seligman S, Tronick E, editors. Infant and early childhood mental health. Arlington: American Psychiatric Association; 2014.

14. Greenspan S, Wieder S. Relationship-based early intervention approach to autistic spectrum disorders: the DIR model. In: Amaral DG, Dawson $\mathrm{G}$, Geschwind $\mathrm{DH}$, editors. Autism spectrum disorders. New York: Oxford University Press; 2011. p. 1068-180.

15. Gonçalves MJ, Caldeira da Silva P. A classificação diagnóstica das perturbações da saúde mental da primeira infância: uma experiência clínica. Análise Psicol. 2003;1:13-21.

16. Barton M, Robins D, Jashar D, Brennan L, Fein D. Sensitivity and specificity of proposed DSM-5 criteria for autism spectrum disorder in toddlers. J Autism Dev Disord. 2013;43:1184-95.

17. Diagnostic classification of mental health and developmental disorders of infancy and early childhood: revised edition (DC:0-5). Washington: Zerotothree; 2017.

18. American Psychiatric Association. Manual de Diagnóstico e Estatística das Perturbações Mentais DSM 5. $5^{\mathrm{a}}$ ed. Lisboa: Climepsi Editores; 2014.
19. World Health Organization. The ICD-10 classification of mental and behavioural disorders: diagnostic criteria for research. Geneva: WHO; 1993.

20. National Institute for Health and Care Excellence. Autism: the management and support of children and young people on the autism spectrum. London: National Institute for Health and Care Excellence; 2013.

21. Fuentes-Biggi J, Ferrari-Arroyo MJ, Boada-Muñoz L, Touriño-Aguilera $E$, Artigas-Pallarés $J$, Belinchón-Carmona $M$, et al. Guía de buena práctica para el tratamiento de los trastornos del espectro autista. Rev Neurol. 2006;43:425-38.

22. Scottish Intercollegiate Guidelines Network. Diagnosis and clinical interventions for children and young people with autism spectrum disorders: a national clinical guideline. SIGN 145. Edinburgh: Scottish Intercollegiate Guidelines Network; 2016.

23. National Institute for Health and Care Excellence. Autism spectrum disorder in under 19s: recognition, referral and diagnosis. Clinical guideline. 2011;128. [accessed $2019 \mathrm{dez} 12$ ] Available at: https://www. nice.org.uk/guidance/cg128/resources/autism-spectrum-disorder-inunder-19s-recognition-referral-and-diagnosis-pdf-35109456621253.

24. Parr J, Woodbury-Smith M. Autism spectrum disorder. BMJ Best Practices. 2017;59-83.

25. Hayes J, Ford T, Rafeeque H, Russell G. Clinical practice guidelines for diagnosis of autism spectrum disorder in adults and children in the UK: a narrative review. BMC Psychiatry. 2018;43:222-47.

26. Direção Geral da Saúde. Abordagem diagnóstica e intervenção na perturbação do espetro do autismo em idade pediátrica e no adulto. Norma da Direção Geral da Saúde (DGS) n002/2019; Lisboa: DGS; 2019.

27. Caldeira da Silva P, Eira C, Pombo J, Silva AP, Corrêa da Silva L, Martins F, et al. Programa clínico para o tratamento das perturbações da relação e da comunicação, baseado no modelo D.I.R. Análise Psicol. 2003;1:31-40.

28. Ligsay A, Bain J, Veenstra-VanderWeele, Hagerman R. Pediatric and neurological assessment and targeted treatments. In: Hollander $\mathrm{E}$, Hagerman R, Fein D, editors. Autism spectrum disorders. Washington: American Psychiatric Association; 2018. p. 143-74.

29. Hyman S, Levy S, Myers S, Council on Children with Disabilities, Section on Developmental and Behavior Pediatrics. Identification, evaluation, and management of children with autism spectrum disorder. Pediatrics. 2020;145:e20193447.

30. Robins D, Fein D, Barton M, Green J. The modified checklist for autism in toddlers: an initial study investigating the early detection of autism and pervasive developmental disorders. J Autism Dev Disord. 2001;31:131.

31. Robins D, Casagrande K, Barton M, Chen C, Dumont-Mathieu T, Fein D. Validation of the modified checklist for autism in toddlers, revised with follow-up (M-CHAT-R/F). Pediatrics. 2013;133:37-45.

32. Direção Geral da Saúde. Programa Nacional de Saúde Infantil e Juvenil. Lisboa: DGS; 2013.

33. Silveira H, Martins A, Ferreira A, Melo L, Varela R. Conhecer Arroios. Lisboa: Instituto Superior Técnico, Junta de Freguesia de Arroios; 2017.

34. Instituto Superior de Psicologia Aplicada. Análise Psicológica 20 anos da Unidade da Primeira Infância. Série XXI. Lisboa: ISPA; 2003. 